\title{
Acanthamoeba Keratitis: Different Surgical Approaches
}

\author{
${ }^{1}$ Mukharram Bikbov, ${ }^{2}$ Valentina Surkova, ${ }^{3} E$ min Usubov
}

\begin{abstract}
The features of acanthamoeba keratitis (AK) progression, clinical cases, and results of early and delayed penetrating keratoplasty as the main method of severe AK treatment are presented. It is described as two clinical cases with different surgical approaches: Case 1 - delayed keratoplasty after remission and case 2 - early keratoplasty during a severe flare up of the disease.

In the 1st case the keratitis led to the development of chronic keratouveitis, secondary glaucoma, complicated cataract, and vision loss. The received keratoplasty was of only anatomic effect. In the 2 nd case early keratoplasty allowed avoiding secondary complications and retaining a satisfactory visual acuity along with avoiding reoperations.
\end{abstract}

Keywords: Acanthamoeba keratitis, Keratitis, Penetrating keratoplasty.

How to cite this article: Bikbov M, Surkova V, Usubov E. Acanthamoeba Keratitis: Different Surgical Approaches. Int J Kerat Ect Cor Dis 2016;5(2):77-80.

Source of support: Nil

Conflict of interest: None

\section{INTRODUCTION}

Acanthamoeba keratitis (AK) is inflammation of the cornea caused by a protozoan - acanthamoeba. ${ }^{1}$ The disease occurs with higher incidence in contact lens (CL) wearers, when an infectious agent known as acanthamoeba gets under CL. ${ }^{2-4}$ There were described cases of AK after Lasik. ${ }^{5,6}$ Microtraumas of cornea epithelium and contact with contaminated environmental sources are risk factors for acanthamoeba invading the cornea. $^{3}$

Long lifecycle of acanthamoeba, including a cyst stage, causes keratitis recurrence for a long period of time. A severe pain in the eye typical for AK at the early stages of the disease is explained by the appearance of subepithelial infiltrates and nerve fibers damage in the superficial, most

\footnotetext{
${ }^{1}$ Chief, ${ }^{2,3}$ Leading Research Scientist

${ }^{1}$ Department of Management, Ufa Eye Research Institute, Ufa Bashkortostan, Russian Federation

${ }^{2,3}$ Department of Cornea and Lens Surgery, Ufa Eye Research Institute, Ufa, Bashkortostan, Russian Federation

Corresponding Author: Emin Usubov, Leading Research Scientist, Department of Cornea and Lens Surgery, Ufa Eye Research Institute, Ufa, Bashkortostan, Russian Federation Phone: +73472735108, e-mail: ullss@yandex.ru
}

sensitive layer of the cornea. The diagnosis deemed to be complete only when cysts are detected in the material taken from the cornea and its agar inoculation, smears of CLs, and their cases.

The AK conservative treatment is conducted by antiseptics use. The most effective treatment to combat cysts is chlorhexidine $0.02 \%$, which is prepared ex. temporae. Polyhexamethylene $0.02 \%$ as part of the solution is used for CLs disinfection, and can be used off-label in $\mathrm{AK}$.

Clinical cases provided testify to the difficulties in the AK diagnosis and showed features of the disease.

\section{CASE REPORTS}

\section{Case 1}

A 32-year-old male patient was hospitalized to our clinic in June 2011. On examination he had: OU - moderate to severe myopia, Right eye (OD) - keratitis of unknown etiology. Visual acuity: OD - 0.09, Best corrected visual aquity $(\mathrm{BCVA})-4.0$ diopters $=0.6$; Left eye $(\mathrm{OS})$ - healthy, visual acuity $-0.1, B C V A-4.0$ diopter $=1.0$. The patient was a soft CL wearer for over 6 years because of moderate to severe myopia. One day, without any apparent reason, he had symptoms of blepharospasm, photophobia, epiphora, severe pain, chemosis, and reduced visual acuity in the right eye.

Pathologic microflora was not found in a smear and the material was sent for culture from the OD conjunctiva. The scrape showed misshapen epithelial cells and a large number of lymphocytes. Epithelial and subepithelial corneal infiltrates, sometimes confluent, like dendritic herpetic keratitis were revealed along with severe combined injection of the right eye. Anti-inflammatory, antiherpetic, and symptomatic treatment in connection with suspected viral origin of keratitis was done, which led to the improvement in symptoms. Within 3 months periods, improvement and recurrence of right eye keratitis alternated. The disease recurrence with flare up periods put the acanthamoeba etiology suspecting on top of our diagnosis, but acanthamoeba results were negative in scrapes, in material obtained at the corneal biopsy, and in agar inoculation.

In October 2011, during a re-treatment of the patient in the clinic a flare up occurred. A deep ring infiltrate with a diameter of 7 to $8 \mathrm{~mm}$ was observed in the corneal stroma 
OD, iridocyclitis, hypertension, uncorrected visual acuity reduced to 0.05 ; left eye was healthy; best corrected visual acuity was 1.0. By confocal microscopy, acanthamoeba cysts were revealed in the infiltrate of corneal stroma OD and AK was diagnosed.

Due to disease progression, descemetocele, and corneal perforation (Fig. 1A), the patient was hospitalized to the Eye Institute (Moscow), where he underwent a subtotal penetrating keratoplasty. Ahmed valve was implanted (Fig. 1B). The inflammatory process was stopped. A postoperative long-term treatment included the use of symptomatic agents, corticosteroids, nonsteroidal antiinflammatory drugs, selective immunosuppressive drugs, antihypertensive, and regenerative medication. One year after the surgery an opaque engraftment was marked, hypertension despite the topical administration of hypotensive drugs, visual acuity - counting fingers near the face.

In the following years, due to graft $v$ s host disease, the patient twice underwent bio-covering of cadaver cornea with scleral rim to improve a regeneration of the cornea. The bio-cover, which acted as therapeutic CL, was rejected after 9 days post surgery (Fig. 1C). Then a short-term improvement occurred.

The patient was repeatedly admitted to our clinic in November 2014 for emergency indications due to maladjustment of the corneal bio-cover. It was gently removed. Degeneration of the previously transplanted penetrating graft with a crater defect $4 \times 5 \mathrm{~mm}$ in the center, was revealed. The patient underwent repeated penetrating keratoplasty of $8.5 \mathrm{~mm}$ diameter with a reconstruction of the anterior part of the eye, including synechotomy, partial iridectomy, removal of the blurred lens, reposition, and irrigation of Ahmed valve tube. The surgery was completed with overlaying 18 interrupted nylon sutures.

In the postoperative period (within 5 months), the eye was quiet, the graft was clear, interrupted sutures secured the graft well in a trephine opening (Fig. 1D), deep anterior chamber, irregularly shaped pupil, quiet iris, aphakia, red reflex of the fundus, glaucoma chronic optic neuropathy, and atrophy of the optic nerve. Visual acuity was " 0 ".
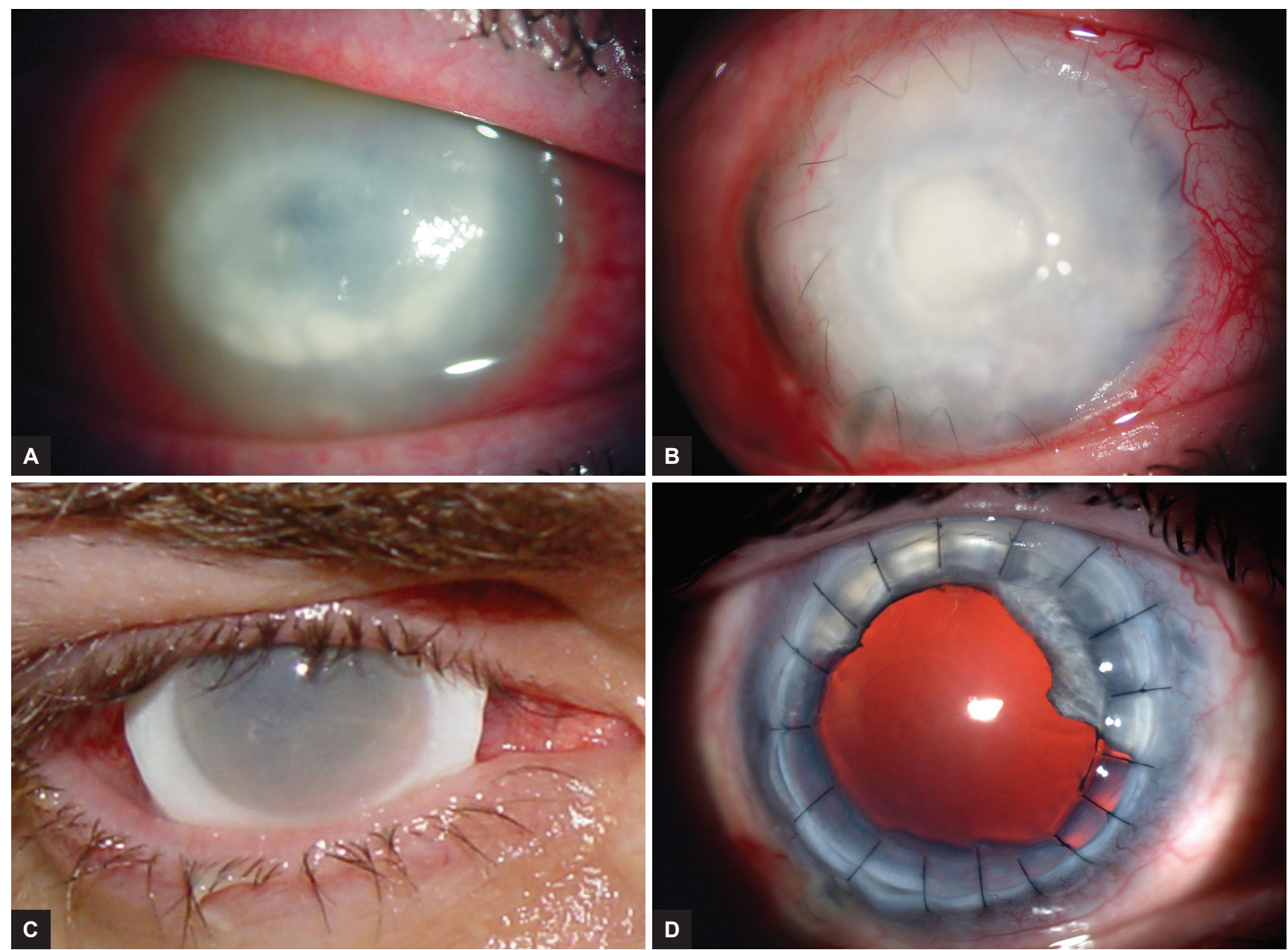

Figs 1A to D: Acanthamoeba keratitis: (A) A flare up of the disease: a ring-shaped infiltrate in the cornea; (B) a state after the 1 st penetrating keratoplasty and Ahmed valve implantation (at 7 clock hours); (C) a temporary bio-cover with corneascleral flap in 3.5 years after the 1st penetrating keratoplasty; and (D) a state after re-penetrating keratoplasty with a reconstruction of the anterior segment of the eye (case 1) 


\section{Case 2}

A 36-year-old female patient was admitted to our hospital with complaints of eye redness, foreign body sensation, and drastic visual acuity reduction in the left eye. Since childhood, she has had a diagnosis of moderate to severe myopia and has been a CL wearer for 8 years. Periodically, she failed to comply with CL hygiene and terms of their use. At primary presentation in an out-patient clinic, she complained of pain, photophobia, and reduced visual acuity.

The patient was started on topical instillations of levofloxacin and ophtalmoferon. However, no improvement was observed: After 3 days of starting the treatment, the pain, epiphora increased and the best corrected visual acuity was 0.01 . On the 7 th day of the disease, the patient was directed to our clinic. On examination at our clinic, her diagnosis was as follows: OU - moderate to severe myopia. OS - keratitis of unknown etiology. Visual acuity -0.005 .

Physical examination: OD - quiet. OS - sharp conjunctival hyperemia, chemosis, swollen cornea in the upper sector, infiltrate $5 \times 4 \mathrm{~mm}$ with perifocal edema, corneal thinning in the center of the lesion, descemetocele $1 \times 1 \mathrm{~mm}$ (Fig. 2). Confocal microscopy identified subepithelial, stromal, hyperechoic acanthamoeba cysts. It was recommended as a tectonic penetrating keratoplasty.

Antiseptic, antibacterial, and anti-inflammatory treatment showed no signs of improvement. Due to a complicated confirmed diagnosis - AK and corneal perforation threat - a penetrating keratoplasty was performed with therapeutic and visual rehabilitation purposes on the $3 \mathrm{rd}$ day after her presentation to the clinic.

A penetrating keratoplasty was performed according to standard procedures with general intravenous anesthesia. The donor graft size was $7 \mathrm{~mm}$ and the host area was $6.75 \mathrm{~mm}$. The graft was secured with uninterrupted

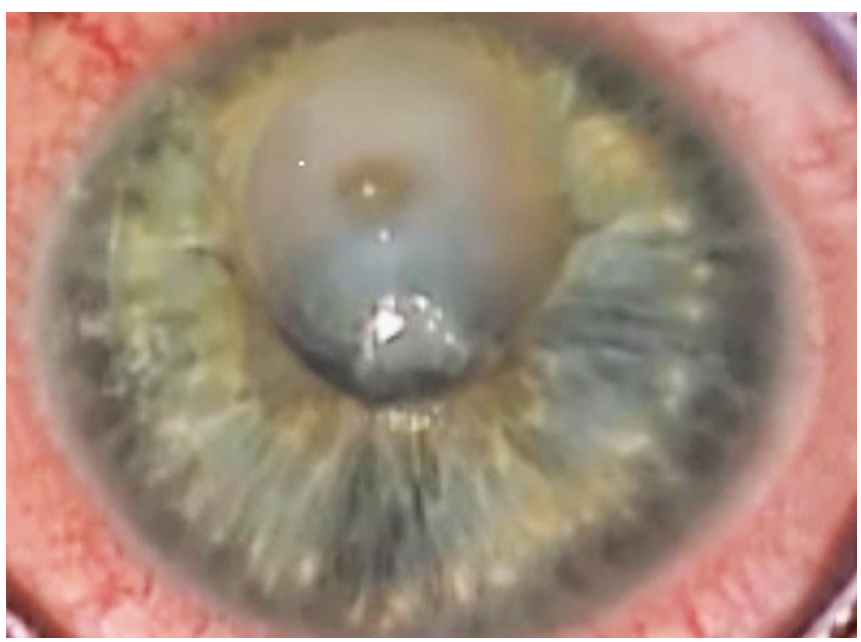

Fig. 2: Biomicroscopic corneal examination before surgery suture. After the surgery, anti-inflammatory and immunosuppressive treatment was prescribed. Antiprotozoal treatment was continued after the surgery, and cytostatic drug cyclosporin A was administered topically per 1 drop 2 times a day, along with systemic antibiotics and antiprotozoal drugs - ceftriaxone and metronidazole, corticosteroids - dexamethasone that was tapered over then.

On the 1st day after surgery, the patient complained of slight photophobia, epiphora. Visual acuity increased to 0.09 . On physical examination, the left eye was moderately irritated, graft was slightly edematous, sutures well fixed, beginning of corneal epithelialization at the periphery of the graft was noticed. On the 4 th day, after the surgery epithelization of the cornea completed, graft transparency recovered, rims adapted. Visual acuity of the operated eye was 0.2 . One month after the surgery and treatment with topical steroids, cyclosporin A, $20 \%$ solcoseryl gel, improvement in uncorrected visual acuity to 0.4 was observed; best corrected visual acuity $\mathrm{sph}+2.5 \mathrm{D}$ was 0.5 , and further remained stable. The graft was clear and sutures were well fixed (Fig. 3). According to confocal microscopy, the endothelial cell density in the central part of the graft was $1874 \pm 25$ cells $/ \mathrm{mm}^{2}$, and extracellular matrix was completely transparent.

\section{DISCUSSION}

Establishing etiology of the disease represents certain difficulties as not always possible to detect cysts. Careful history taking, follow-up of keratitis clinical course, use of confocal microscopy, which allows detecting acanthamoeba cysts in the cornea in vivo will help to set a diagnosis. In case of severe keratopathy, a lamellar or penetrating keratoplasty is the single approach to AK treatment. ${ }^{7}$ The retrospective studies by Ross et $\mathrm{al}^{8}$ are of interest, who analyzed data of 116 patients with

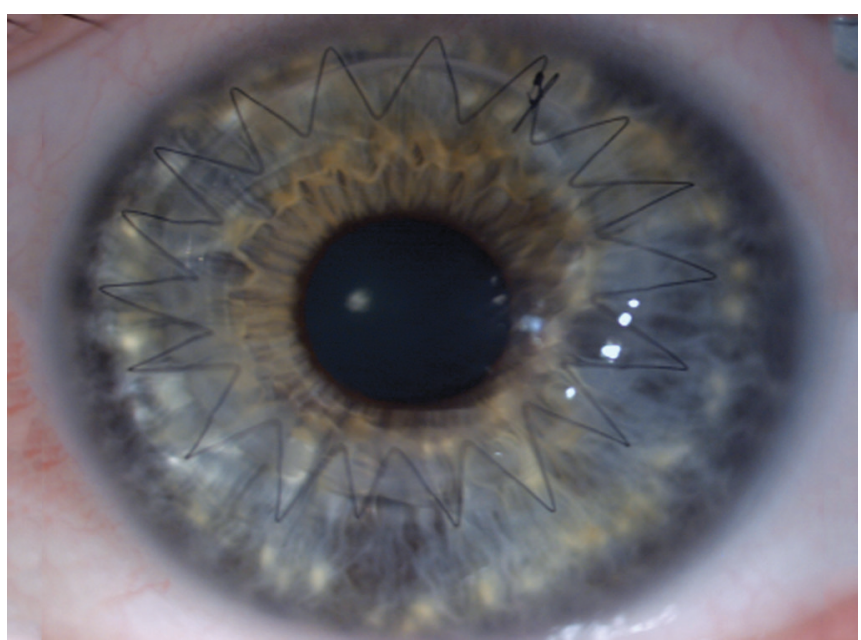

Fig. 3: Biomicroscopic corneal examination 1 month after penetrating keratoplasty 
AK during 2008-2011. The authors found that $93.3 \%$ of patients with $A K$ were $C L$ wearers, and an average period from onset of symptoms to diagnosis took 27 days. The keratoplasty conducted in 27 of 81 patients showed that the adverse outcome of surgery was associated with senior age of patients and lesion of deep corneal layers, especially in the presence of ring-infiltrate of the last. ${ }^{7}$

Untimely diagnosis and delayed treatment of patients with AK, contribute to corneal perforation with involvement of deep structures of the eye in the inflammatory process, which quite often leads to eye enucleation. ${ }^{4}$

The provided clinical cases testify the connection of the disease with CL wearing or eye injury, AK diagnostic difficulties, clinical features, and a long period of rehabilitation. Due to the similarity of the disease beginning with herpes simplex infection, specialists often start treatment with antiherpethetical drugs and in this connection, first of all a differential diagnostics is required. The surgical approach in the treatment of this disease may differ depending on the severity and area of corneal lesions. The cases presented have shown that early keratoplasty as a definitive therapy may be the most effective in terms of functionality, although the risk of the disease recurrence retains.

Prognosis for AK depends on timely diagnosis and appropriate treatment, timely subsequent keratoplasty, and further balanced treatment with corticosteroids in combination with selective immunosuppressive drugs. Keratoplasty is the single method of severe AK definitive therapy, which preserves the eye and improves visual acuity.

Acanthamoeba keratitis should be suspected in any patient, who uses CLs, especially with remitted keratitis. Patients should be warned about seriousness of the disease, its recurrence with vision loss, and necessity for follow-up by ophthalmologists. Early treatment can limit the affected area, which improves the efficiency of surgical treatment and preserves the functions of the eye.

\section{CONCLUSION}

The two cases presented in this study demonstrate that treatment of AK with early surgical approach using penetrating subtotal keratoplasty, which may be efficient in achieving satisfactory functional results.

\section{REFERENCES}

1. Thebpatiphat N, Hammersmith KM, Racha FN, Rapuano CJ, Ayres BD, Laibson PR, Eagle RC Jr, Cohen EJ. Acanthamoeba keratitis: a parasite on the rise. Cornea 2007 Jul;26(6):701-706.

2. Kaur H, Maquire H, Salomao RD, Cameron JD. Rapid progression of amebic keratitis 1 week after corneal trauma and 1 year after Lasik. Cornea 2007 Feb;26(2):212-214.

3. Dart JK, Saw VP, Kilvington S. Acanthamoeba keratitis: diagnosis and treatment update 2009. Am J Ophthalmol 2009 Oct;148(4):487-499.

4. Hau SC, Dart JK, Vesaluoma M, Parmar DN, Claerhout I, Bibi K, Larkin DF. Diagnostic accuracy of microbial keratitis with in vivo scanning laser confocal microscopy. Br J Ophthalmol 2010 Aug;94(8):982-987.

5. Clarke DW, Niederkorn JY. The pathophysiology of Acanthamoeba keratitis. Trends Parasitol 2006 Apr;22(4):175-180.

6. Marciano-Cabral F, Cabral G. Acanthamoeba spp. as agents of disease in humans. Clin Microbiol Rev 2003 Apr;16(2): 273-307.

7. Tu EY, Joslin CE, Sugar J, Booton GC, Shoff ME, Fuerst PA. The relative value of confocal microscopy and superficial corneal scrapings in the diagnosis of Acanthamoeba keratitis. Cornea 2008 Aug;27(7):764-772.

8. Ross J, Roy SL, Mathers WD, Ritterband DC, Yoder JS, Ayers T. et al. Clinical characteristics of Acanthamoemba Keratitis Infections in 28 states, 2008 to 2011. Cornea 2014;33(2):161-168. 